\title{
EDUCAÇÃO EM SAÚDE
}

\section{E O APRIMORAMENTO DE}

NOVOS CONHECIMENTOS

\section{Organizador:}

Pablo Augusto Gurgel de Sousa

VOLUME 1

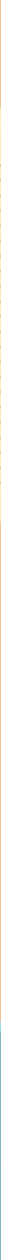




\section{EDUCAÇÃO EM SAÚDE}

\section{E O APRIMORAMENTO DE NOVOS CONHECIMENTOS}

Organizador:

Pablo Augusto Gurgel de Sousa

\section{VOLUME 1}

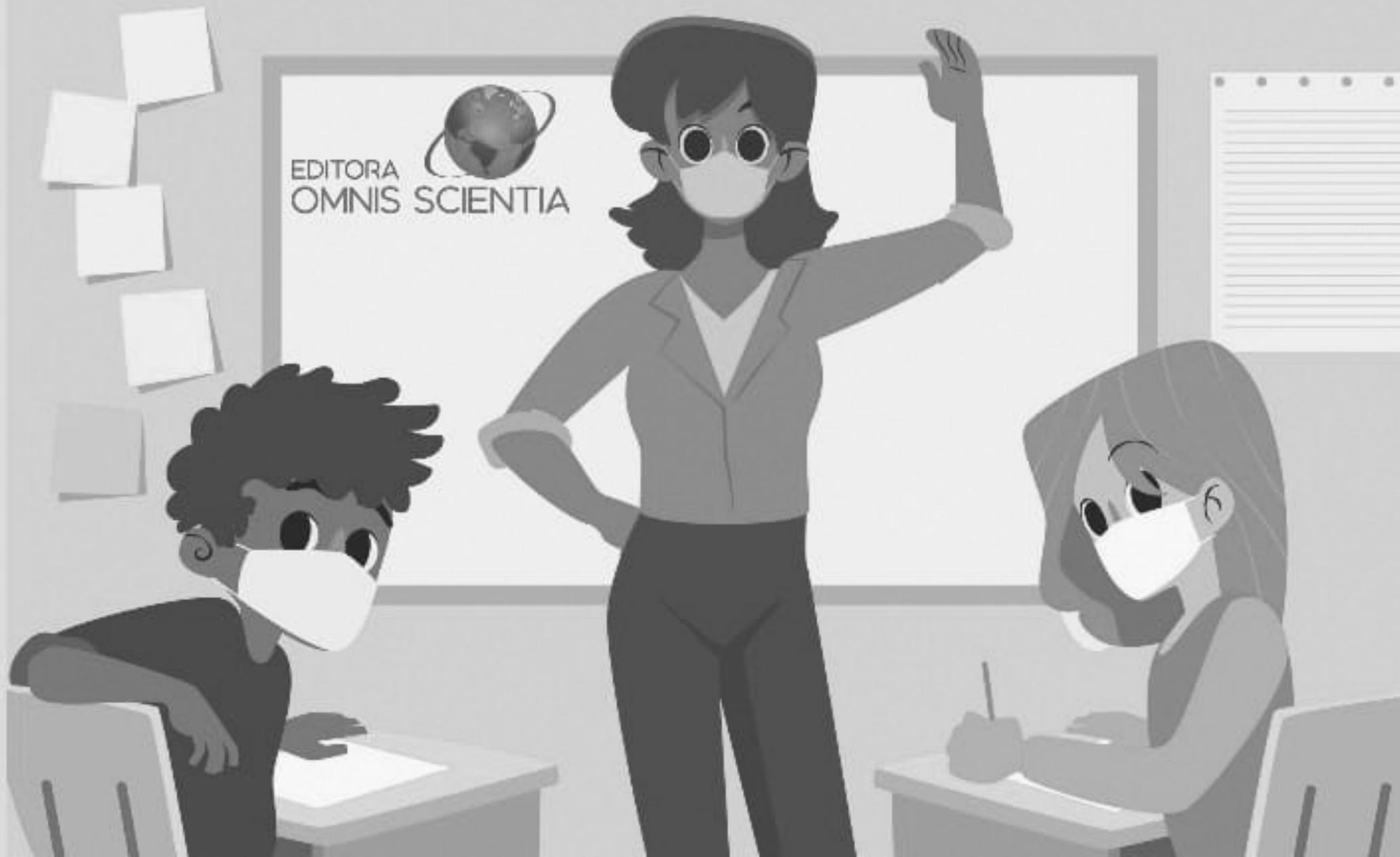


Editora Omnis Scientia

EDUCAÇÃO EM SAÚDE E O APRIMORAMENTO DE NOVOS CONHECIMENTOS

Volume 1

$1^{a}$ Edição

TRIUNFO - PE 


\section{Editor-Chefe}

Me. Daniel Luís Viana Cruz

Organizador

Me Pablo Augusto Gurgel de Sousa (Mestre em Psicobiologia)

\section{Conselho Editorial}

Dr. Cássio Brancaleone

Dr. Marcelo Luiz Bezerra da Silva

Dra. Pauliana Valéria Machado Galvão

Dr. Plínio Pereira Gomes Júnior

Dr. Walter Santos Evangelista Júnior

Dr. Wendel José Teles Pontes

Editores de Área - Ciências da Saúde

Dra. Camyla Rocha de Carvalho Guedine

Dra. Cristieli Sérgio de Menezes Oliveira

Dr. Leandro dos Santos

Dr. Hugo Barbosa do Nascimento

Dr. Marcio Luiz Lima Taga

Dra. Pauliana Valéria Machado Galvão

\section{Assistente Editorial}

Thialla Larangeira Amorim

Imagem de Capa

Freepik

\section{Edição de Arte}

Vileide Vitória Larangeira Amorim

\section{Revisão}

Os autores

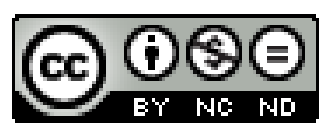

Este trabalho está licenciado com uma Licença Creative Commons - AtribuiçãoNãoComercial-SemDerivações 4.0 Internacional.

O conteúdo abordado nos artigos, seus dados em sua forma, correção e confiabilidade são de responsabilidade exclusiva dos autores. 


\title{
Dados Internacionais de Catalogação na Publicação (CIP)
} (eDOC BRASIL, Belo Horizonte/MG)

E24 Educação em saúde e o aprimoramento de novos conhecimentos [livro eletrônico] / Organizador Pablo Augusto Gurgel de Sousa. Triunfo, PE: Omnis Scientia, 2021.

145 p. : il.

Formato: PDF

Requisitos de sistema: Adobe Acrobat Reader

Modo de acesso: World Wide Web

Inclui bibliografia

ISBN 978-65-88958-61-2

DOI 10.47094/978-65-88958-61-2

1. Educação sanitária. 2. Saúde pública. 3. Qualidade de vida.

I. Sousa, Pablo Augusto Gurgel de.

CDD 362.1

Elaborado por Maurício Amormino Júnior - CRB6/2422

\author{
Editora Omnis Scientia \\ Triunfo - Pernambuco - Brasil \\ Telefone: +55 (87) 99656-3565 \\ editoraomnisscientia.com.br \\ contato@editoraomnisscientia.com.br
}




\section{PREFÁCIO}

Saudações prezado (a) leitor (a),

Em seu livro Dez Bilhões, publicado em 2013, o professor Stephen Emmott, de Oxford, indagou que "há 10.000 anos éramos apenas um milhão. Em 1800, faz pouco mais de 200 anos, já éramos um bilhão. Há 50, por volta de 1960, chegamos a 3,5 bilhões. Atualmente, superamos 7,5 bilhões”. Mais precisamente, segundo dados do novo relatório do Population Reference Bureau (PRB), somos cerca de 7,8 bilhões de seres humanos habitando este planeta no momento. Não obstante à visão apocalíptica deste panorama, a que se deve tamanha progressão geométrica?

Consenso entre historiadores e estudiosos da demografia humana, muito mais do que abandonar o modo de vida nômade, as descobertas e avanços da área médica foram fundamentais para que os séculos XX e XXI registrassem um elevado crescimento populacional. É notável que, a partir desse período, se consolidou e se difundiu a importância da pesquisa em saúde, não só com o objetivo de sanar doenças, mas também de prevenção e promoção à saúde, provendo ao indivíduo e à sociedade meios para a melhoria da qualidade de vida.

Nesta perspectiva, sabendo que o conhecimento científico é muito valioso, principalmente em um cenário pandêmico causado pelo vírus Sars-CoV-2, a Editora Omnis Scientia nos abrilhanta com o livro Educação em Saúde e o aprimoramento de novos conhecimentos. Por meio de um compilado de artigos, este constructo evidencia a importância do papel dos profissionais de saúde como divulgadores científicos, seja em pesquisas teóricas, aplicadas, de inovação tecnológica ou mesmo relatos de experiências, combatendo a cultura da desinformação, auxiliando a promoção de políticas públicas efetivas e refletindo sobre as nossas ações perante a sociedade como um todo.

Ademais, esta publicação surge em circunstância significativa como forma de promover o avanço, ainda mais expressivo, do processo de inserção do Brasil no patamar dos grandes centros científicos do mundo. Essa iniciativa, portanto, deve ser celebrada, além do mais, pela disseminação do conhecimento científico em educação em saúde, adequado em qualidade e momento oportuno, primordial para promoção do bem-estar populacional.

Por fim, em nossos livros, selecionamos um dos capítulos para premiação como forma de incentivo para os autores, e entre os excelentes trabalhos selecionados para compor este livro, o premiado foi o capítulo 2, intitulado "Residência multiprofissional em Saúde da Família e as contribuições para a interprofissionalidade e a formação do Assistente Social". Por meio de relato de experiência vivenciada pela residente de serviço social, o trabalho nos traz reflexões sobre as contribuições do programa de residência no processo de aprendizagem e qualificação profissional, bem como, para as ações multiprofissionais de educação em saúde, desenvolvidas em conjunto com os residentes de diversas áreas da saúde. 


\section{SUMÁRIO}

CAPÍTULO 1 .14

ENFRENTAMENTO À COVID-19 PELA EQUIPE MULTIPROFISSIONAL NA ATENÇÃO BÁSICA E SAÚDE DA FAMÍLIA

Patrícia Fernanda Faccio

Ântony Eliel Andrade da Silva

Brenda Fernanda Guedes

José Filipe da Silva

Kristine Kelly de Albuquerque

Maria Daniele da Silva

Marianne de Araújo Mendes

Mércia Fernanda Melo da Silva

Taise Maria da Costa

João Paulo Maciel Cavalcanti de Albuquerque

DOI: 10.47094/978-65-88958-61-2/14-20

CAPÍTULO 2

RESIDÊNCIA MULTIPROFISSIONAL EM SAÚDE DA FAMÍLIA: INTERPROFISSIONALIDADE E A FORMAÇÃO DO ASSISTENTE SOCIAL

Rafaela Zulmira de Oliveira Moraes

Christiani Cassoli Bortoloto Lopes

Andréia Santina Seubert Dalferth

Évelyn Farias

Estefany Bahert

Pedro Henrique de Carli

Maria Nazaré Murilho

Isabela Cristina Mannes

Danieli Cristina Scalco 
Felipe Gustavo de Bastiani

Yasmin Luisa Dengo Lombardo

Gisielli Jovenilia Polidorio Alievi

DOI: 10.47094/978-65-88958-61-2/21-37

CAPÍTULO 3. .38

AÇÃO EDUCATIVA COMO FERRAMENTA PARA PREVENÇÃO DO CÂNCER DE MAMA EM UMA UNIDADE DE ATENÇÃO PRIMÁRIA À SAÚDE

Matheus Gomes Andrade

Dilene Fontinele Catunda Melo

Maria Larysse Muniz Pereira

Lurdiane Gabriel Pereira

Maria Aparecida Melo Morais

Glória Vanessa de Araújo Silva Sousa

Jesus Carlos Eduardo de Paiva Avelino

Fernanda Alália Braz de Sousa

Maria das Graças Teodosio Dias

Viceni Almeida Ludgero

Rosângela Souza Cavalcante

Francisca Nellie de Paula Melo

DOI: 10.47094/978-65-88958-61-2/38-44

CAPÍTULO 4 .45

TRATAMENTO INTRALESIONAL DE LEISHMANIOSE CUTÂNEA LOCALIZADA (LCL) EM UNIDADE BÁSICA DE SAÚDE DURANTE PANDEMIA

Sarah Ramany Faria Salmeron

Daliany Santos

Adrielly Sousa Guimarães

Lucas Salvador Pereira 
CAPÍTULO 5. .51

ESTRATÉGIAS DE EDUCAÇÃO EM SAÚdE PARA CRIANÇAS EM TEMPOS DE PANDEMIA: RELATO DE EXPERIÊNCIA

Mirelly Shatilla Misquita Tavares

Clara de Sousa Rodrigues

Anna Beatriz de Almeida Gomes Sousa

Mikaelly Teixeira Alves

Naylton Morais Dias

Dannilo Dias Soares

Viceni Almeida Ludgero

Wagner da Costa Bezzerra

Fernanda Alália Braz de Sousa

Carlos Alberto Cavalcante de Lima

Mariane Pereira da Luz Melo

Samara Lais Silva Ferreira

Francisca de Fatima dos Santos Freire

DOI: 10.47094/978-65-88958-61-2/51-61

CAPÍTULO 6 .62

ATUAÇÃODAENFERMEIRANAEQUIPE MULTIPROFISSIONALNOTRATAMENTO DE CÂNCER: RELATO DE EXPERIÊNCIA

Carla Walburga da Silva Braga

DOI: 10.47094/978-65-88958-61-2/62-69 
EDUCAÇÃO EM SAÚdE BUCAL COMO ESTRATÉGIA DE PREVENÇÃO À CÁRIE DENTÁRIA NA INFÂNCIA

Lara Pepita de Souza Oliveira

Jardel dos Santos Silva

Jefter Haad Ruiz da Silva

Esaú Lucas Nascimento Tavares

Ivana Caroline de Souza Marinho Araújo

Luca Ramon da Silva Lima

Ivete Castro de Souza

Kerolayne Sena de Sousa Santos

Erika Akiko Moura Shiota

Dina Birman

Cristiane Maria Brasil Leal

Diego Ferreira Regalado

DOI: 10.47094/978-65-88958-61-2/70-79

CAPÍTULO 8. 80

\section{TÓPICOS RELEVANTES RELACIONADOS À SAÚDE DA MULHER}

Lília Barroso Cipriano de Oliveira

Rebeca Barroso Cipriano de Oliveira

Regizeuda Ponte Aguiar

DOI: 10.47094/978-65-88958-61-2/80-86

CAPÍTULO 9 .87

ESTRATÉGIA PARA EDUCAÇÃO EM SAÚDE EM TEMPOS DE PANDEMIA: RODA DE CONVERSA VIRTUAL SOBRE APLV

Ludmylla Rolim de Albuquerque

Mayara Vieira Rodrigues 
Bruna Ramalho Nogueira Diniz

Maria Luíza Formiga Barros Batista

Taynara Souza Santos

Núbia Kelly Rodrigues Ribeiro

Ideltônio José Feitosa Barbosa

DOI: 10.47094/978-65-88958-61-2/87-94

CAPÍTULO 10 .95

CONHECIMENTO DOS UNIVERSITÁRIOS DO SEXO MASCULINO SOBRE O USO DO ANTICONCEPCIONAL DE EMERGÊNCIA

Laryssa Bezerra Silva

Nathália Lima de Pontes

Graziani Izidoro Ferreira

Fernanda Souza e Silva Garcia

DOI: 10.47094/978-65-88958-61-2/95-101

CAPÍTULO 11

SAÚdE E TECNOLOGIA: A PERCEPÇÃO DE JOVENS RURAIS ACERCA DA TELESAÚDE NO CUIDADO EM PSICOTERAPIA

Isadora Ribas Strojarki

Marcelo Moreira César

Thalia Brites Muniz

Ana Carolina Ferraz

Dawid Da Silva Vargas

DOI: 10.47094/978-65-88958-61-2/102-116 
TELERREABILITAÇÃO COMO RECURSO FISIOTERAPÊUTICO NAATENÇÃO BÁSICA

FRENTE À PANDEMIA DE COVID - 19

Patrícia Fernanda Faccio

Alex Lira do Nascimento e Silva

Elaine Ferreira Silva

Samuel Cézar Alexandre Silva

Mércia Fernanda Melo da Silva

Giuliane Diógenes Norberto da Silva

Jullia Carolyne Rosa Cordeiro de Lima

Tatianny dos Santos Cassiano

Paula Drielly de Melo Ribeiro

Soraya Santos Alves Barbosa

João Paulo Maciel Cavalcanti de Albuquerque

DOI: 10.47094/978-65-88958-61-2 /117-125

CAPÍTULO 13.

USO DO INSTAGRAM COMO FERRAMENTA DE EDUCAÇÃO EM SAÚDE SOBRE APLV DURANTE A PANDEMIA DO COVID-19

Emanuel de Oliveira Colombo

Laysa Bianca Gomes de Lima

Abiel Reyfe da Silva Canuto

Núbia Kelly Rodrigues Ribeiro

Ideltônio José Feitosa Barbosa

DOI: 10.47094/978-65-88958-61-2/126-133 
CAPÍTULO 14.

VIABILIDADE DOS APLICATIVOS m-HEALTH PARA PACIENTES COM DOENÇAS PULMONARES CRÔNICAS NO SISTEMA PÚBLICO DE SAÚDE DO DISTRITO FEDERAL

Heloisa Glass

Gabriel Cordeiro Schimidt

Igor Louza Pereira

Paulo Henrique de Ramos Feitosa

DOI: 10.47094/978-65-88958-61-2 /134-141 


\section{AÇÃO EDUCATIVA COMO FERRAMENTA PARA PREVENÇÃO DO CÂNCER DE MAMA EM UMA UNIDADE DE ATENÇÃO PRIMÁRIA À SAÚDE}

\section{Matheus Gomes Andrade';}

Faculdade Princesa do Oeste (FPO), Crateús, Ceará/CE.

http://lattes.cnpq.br/9159202211572002

Dilene Fontinele Catunda Melo';

Faculdade Princesa do Oeste (FPO), Crateús, Ceará/CE.

http://lattes.cnpq.br/5962035812058006

Maria Larysse Muniz Pereira ${ }^{3}$;

Faculdade Princesa do Oeste (FPO), Crateús, Ceará/CE.

http://lattes.cnpq.br/4652657425395867

\section{Lurdiane Gabriel Pereira ${ }^{4}$;}

Faculdade Princesa do Oeste (FPO), Crateús, Ceará/CE.

http://lattes.cnpq.br/0222650819528952

\section{Maria Aparecida Melo Morais ${ }^{5}$;}

Faculdade Princesa do Oeste (FPO), Crateús, Ceará/CE.

http://lattes.cnpq.br/9754154993033273

Glória Vanessa de Araújo Silva Sousa ${ }^{6}$;

Faculdade Princesa do Oeste (FPO), Crateús, Ceará/CE.

http://lattes.cnpq.br/7723625036760730

Jesus Carlos Eduardo de Paiva Avelino ${ }^{7}$;

Faculdade Princesa do Oeste (FPO), Crateús, Ceará/CE.

http://lattes.cnpq.br/6102475556748550

Fernanda Alália Braz de Sousa;

Faculdade Princesa do Oeste (FPO), Crateús, Ceará/CE.

http://lattes.cnpq.br/5713236356265394 


\section{Maria das Graças Teodosio Dias';}

Faculdade Princesa do Oeste (FPO), Crateús, Ceará/CE.

$\underline{\text { http://lattes.cnpq.br/3820168284549275 }}$

\section{Viceni Almeida Ludgero ${ }^{10}$;}

Faculdade Princesa do Oeste (FPO), Crateús, Ceará/CE.

http://lattes.cnpq.br/5108235899781211

\section{Rosângela Souza Cavalcante ${ }^{11}$;}

Faculdade Princesa do Oeste (FPO), Crateús, Ceará/CE.

http://lattes.cnpq.br/3782812914528918

\section{Francisca Nellie de Paula Melo ${ }^{12}$.}

Faculdade Princesa do Oeste (FPO), Crateús, Ceará/CE.

\section{http://lattes.cnpq.br/9409811125591959}

RESUMO: O câncer de mama na população vem sendo considerado um acentuado problema de saúde pública e em evidência na população feminina. Este estudo tem o objetivo de relatar a experiência de acadêmicos do curso de bacharelado em enfermagem em extensão em saúde indígena no município de Crateús, na qual foram utilizados métodos lúdicos a pacientes mulheres indígenas, sobre a prevenção do câncer de mama. No mês de outubro de 2020, o grupo da extensão indígena executou uma educação em saúde com tema: "Prevenção ao Câncer de Mama: Mulher, Seja Você Sua Própria Heroína" na sala de espera de uma unidade de atenção primária de saúde com mulheres indígenas sobre a prevenção do câncer de mama. Utilizou-se de metodologias ativas, a citar: a mama em tecido em um painel para demonstrar sobre os achados fisiológicos e anormais e um espelho para ensinar o autoexame das mamas, e como resultado obteve-se a interação com essas mulheres, a aproximação e respostas para muitas perguntas, e a realização do exame, mesmo com a resistência destas, por não confiar em acadêmicos para a realização do exame. Conseguir essa interação entre pacientes e profissionais Enfermeiros significa uma melhoria da qualidade da assistência nas unidades de atenção à saúde, sendo um dos métodos alternativos que podem ser utilizados para desenvolver práticas de saúde integrais. Os acadêmicos reconhecem a importância dessa vivência para sua formação, tendo a perspectiva de que é um processo fundamental na atuação do profissional enfermeiro e toda equipe multiprofissional dentro do contexto associado a qualificação da saúde da mulher.

PALAVRAS-CHAVE: Câncer de Mama. Educação em Saúde. Enfermagem. 
ABSTRACT: Breast cancer in the population has been considered a major public health problem and in evidence in the female population. This study aims to report the experience of undergraduate nursing students in the extension course in indigenous health in the municipality of Crateús, in which playful methods were used to indigenous women patients on the prevention of breast cancer. In October 2020, the indigenous extension group carried out health education with the theme: "Breast Cancer Prevention: Woman, Be Your Own Heroine" in the waiting room of a primary health care unit with indigenous women about breast cancer prevention. Active methodologies were used, such as: the breast in tissue on a panel to demonstrate the physiological and abnormal findings and a mirror to teach breast self-examination. As a result, the interaction with these women was achieved, the approximation and answers to many questions, and the performance of the exam, even with their resistance, for not trusting academics to perform the exam. Achieving this interaction between patients and nursing professionals means improving the quality of care in health care units, being one of the alternative methods that can be used to develop integral health practices. The students recognize the importance of this experience for their education, with the perspective that it is a fundamental process in the performance of the professional nurse and the entire multiprofessional team within the context associated with the qualification of women's health.

KEY-WORDS: Breast Cancer. Health Education. Nursing.

\section{INTRODUÇÃO}

A saúde da mulher vem recebendo grande destaque no Brasil nos últimos anos, não somente com foco na maternidade, mas sim na mulher como um todo, tendo como consequência a criação a partir do programa de saúde da mulher que inclui ações educativas, preventivas, de diagnóstico, tratamento e recuperação, englobando a assistência à mulher em clínica ginecológica, no pré-natal e puerpério, no climatério, em planejamento familiar, infecções sexualmente transmissíveis, câncer de colo de útero e de mama dentre outros (COUTO et al., 2017).

O câncer de mama na população vem sendo considerado um acentuado problema de saúde pública, em evidência na população feminina. Por se tratar de uma patologia com alta taxa de letalidade e por ter consequências bastante agressivas a saúde seja ela física ou mental da mulher, com uma morbimortalidade característica, a neoplasia de mama tem como fatores de prevenção o rastreamento deste, através da investigação de fatores de risco como o histórico familiar, tabagismo, sobrepeso, sedentarismo e exposição continua à radiação (COUTO et al., 2017).

Essa patologia apresenta como principais sinais e sintomas um nódulo, habitualmente consistente, fixo e indolor; pele da mama avermelhada ou com aspecto de casca de laranja, alterações no mamilo e saída espontânea de líquido de um dos mamilos (INCA, 2020). Assim, os principais 
sintomas e sinais do câncer de mama são nódulos mamários ou axilares, dores nas mamas e alterações na pele cobrindo o seio, com aparência semelhante a casca de laranja (SILVA; RIUL, 2011).

Segundo o INCA (Instituto Nacional do Câncer), a incidência de câncer de mama em 2019 foi de 59,7 mil novos casos, correspondendo a 29,5\% dos cânceres femininos, com exceção do melanoma não canceroso de pele. Em 2016, o número de óbitos femininos por câncer de mama no país foi de 16.069 (INCA, 2019).

INCA (2020), retrata que a prática de exercício físico, alimentação saudável, com manutenção do peso corporal adequado estão associadas diminuição de risco em desenvolver câncer de mama, onde $30 \%$ dos casos podem ser evitados quando são adotados estilos de vida saudáveis. É importante destacar que a amamentação também é considerada um fator protetor.

Dessa forma, o diagnóstico precoce e o rastreamento são os métodos básicos para ajudar na detecção precoce de tumores de mama. O diagnóstico precoce é baseado no método de pessoas sintomáticas, enquanto o rastreamento é projetado para testar e examinar pessoas assintomáticas (OLIVEIRA; CORREIA; FERREIRA, 2017).

Assim, o enfermeiro e a equipe de saúde têm a competência de agir de forma acurada e conjunta para dar conta de demandas, tanto preventivas quanto de promoção da saúde e curativas, bem como educação em saúde na sala de espera para os pacientes das unidades de saúde (OLIVEIRA, 2019).

Este estudo tem o objetivo de relatar a experiência de acadêmicos do curso de bacharelado em enfermagem em um grupo de extensão indígena de uma instituição de ensino superior do sertão de Crateús-CE, na qual foram utilizados métodos lúdicos a pacientes mulheres indígenas sobre a prevenção do câncer de mama.

\section{METODOLOGIA}

Trata-se de um estudo descritivo, do tipo relato de experiência. A pesquisa descritiva é construída a partir da descrição cuidadosa dos fatos e efeitos de uma dada situação, com o objetivo de obter informações sobre questões que foram definidas como em investigação (AUGUSTO, 2013).

No mês de outubro de 2020, o grupo da extensão "PRÁTICAS DE ENFERMAGEM NA PROMOÇÃO Á SAÚDE INDÍGINA" da Faculdade Princesa do Oeste, reuniu-se e executou ações de educação em saúde na sala de espera de uma unidade primária de saúde com 12 pacientes mulheres indígenas sobre a prevenção do câncer de mama, uma patologia neoplásica que vem aumentando consideravelmente as mortalidades e caracterizando-se como um problema de saúde pública.

A experiência relatada foi vivenciada por quatro acadêmicos do curso de Bacharelado de Enfermagem que são membros da extensão acadêmica executada no município de Crateús-CE. A ação ocorreu em uma Unidade de Atenção Primária à Saúde. 
Assim, foi realizada uma educação em saúde com tema: "Prevenção ao Câncer de Mama: Mulher, Seja Você Sua Própria Heroína”, na qual foram utilizadas metodologias ativas, a citar: a mama em tecido em um painel para demonstrar sobre os achados fisiológicos e anormais e um espelho para ensinar o autoexame das mamas, abordando pontos como: o que é a neoplasia de mama, seu quadro clínico, fatores de risco, como prevenir, como é feito o diagnóstico e tratamento, quais exames disponíveis para a identificação e após o diagnóstico o que deve ser feito. Foi apresentado também a rede de atenção e cuidados disponibilizada pelo Sistema Único de Saúde (SUS) para o rastreamento, diagnóstico e tratamento desse câncer.

Nesse contexto, após a educação em saúde com as 12 pacientes indígenas que compareceram na Unidade de Atenção Primária a Saúde as mesmas foram encaminhadas para a sala da enfermeira da unidade para iniciar as consultas ginecológicas bem como o exame preventivo do câncer de colo do útero (PCCU) para coletar uma pequena amostra de células a partir da superfície do colo do útero. A amostra é espalhada sobre uma lâmina de vidro e enviada a um laboratório para análise se há anomalias que podem indicar infecções vaginais ou uterinas.

O encontro dessa ação ocorreu apenas um único dia, uma vez que as pacientes já estavam agendadas para a realizar a consulta preventiva do câncer do colo de útero e mama, uma vez que também é realizado a palpação mamária para identificar se à nódulos ou sinais incomuns.

\section{RESULTADOS E DISCUSSÕES}

O evento foi realizado em cooperação com a equipe da Estratégia de Saúde da Família, a qual se constitui como principal porta de entrada do Sistema Único de Saúde por ter um protocolo de atendimento baseado na descentralização, trabalhando a integralidade, equidade e principalmente universalidade, possibilitando a formação do vínculo profissional e usuário, família, território e fatores determinantes e condicionantes sociais de saúde.

A ação deu início com a apresentação dos discentes e indagação sobre o outubro rosa e porque esse mês é dedicado à prevenção do câncer de mama. As pacientes responderam que: "É $o$ mês destinado a câncer de mama, que é o mais prevalente dentre os outros e o que mais mata, precisa de uma atenção redobrada, pois ainda existem mulheres que não se preocupam com essa enfermidade que ainda é bastante presente na comunidade nos dias de hoje”.

Porém muitas mulheres ainda não conhecem a importância da detecção precoce dessa neoplasia que acomete grande parte da população feminina. Algumas usuárias responderam que: "É apenas um mês para as mulheres fazerem prevenção (exame citopatológico do colo do útero) e prevenir doenças decorrentes a idade".

De forma adicional, as pacientes mulheres passaram a interagir com os acadêmicos de enfermagem no decorrer da ação uma vez que foram surgindo duvidas, além de perderam a timidez por estar a frente de quatro alunos. Com o uso de metodologias ativas para facilitar o processo de ensino-aprendizagem, pode-se perceber um ambiente interativo com relações interpessoais positiva. 
No que tange o uso de metodologias ativas, foram utilizados materiais didáticos a fim de obter o entendimento adequado da população alvo. Foram utilizadas umas mamas confeccionadas de tecido em um painel para que fosse possível a demonstração dos achados normais e anormais, bem como os nódulos ou a pele com aspecto grosso. Em complemento foi explicado sobre a importância da realização do auto exame de mama e possíveis achados, assim foi utilizado um espelho para que as pacientes pudessem ver como deve ser realizado o autoexame.

Com o progredir da apresentação, foram relatados alguns fatores de riscos relacionados ao surgimento do câncer de mama, sendo orientado sobre a importância e eficácia que uma boa alimentação, pratica de exercícios físicos, controle de níveis glicêmicos e pressóricos, consultas periódicas, uso de sutiãs apropriados podem surgir na vida a parti dessas mudanças de hábitos, além da recomendação da mamografia para mulheres a cima de 40 anos de idade.

Assim, a educação em saúde tem como papel a promoção e prevenção de doenças, uma vez que há uma troca de saberes entre o profissional e o paciente, interagido com o individual e cultural possibilitando a produção do cuidado e oferecimento de hábitos e condutas saudáveis.

A conexão com o grupo de usuários é de fundamental, pois os usuários podem compartilhar suas opiniões e conhecimentos prévios e ter oportunidade de tirar dúvidas. Além disso, esse momento abriu espaço para o início da relação entre a equipe e os pacientes, e continuou a manter essa relação no atendimento clínico pessoal.

Os acadêmicos puderam vivenciar a experiência de estar inseridos numa Unidade de Saúde para realizar ações de promoção da saúde de forma positiva, uma vez que a unidade primária de saúde é a porta de entrada e ordenadora de cuidados dos usuários do SUS, ou seja, a uma diversidade imensa de cultura, valores e crenças de cada paciente. Considerando esse contexto, é necessário considerar a singularidade dos diferentes povos e respeitar as manifestações de vontades autônomas no que se diz a respeito quando se vai realizar uma ação com os mesmos.

Diante das colocações mencionadas, a utilização de metodologias ativas na condução das atividades trouxe a melhor compreensão do assunto abordado, e de maneira transformadora, contribuiu para o melhor conhecimento das mulheres.

Logo, o desenvolvimento de estratégias e ações de saúde com foco na educação em saúde transforma-se como um caminho teórico, prático e metodológico de fundamental importância para o fortalecimento das práticas em saúde nos serviços da atenção primária, capaz de superar a fragmentação do cuidado e a compartimentalização dos saberes, resultando numa atenção integral resolutiva, tento através da educação em saúde quanto pela transformação e qualificação do SUS. 


\section{CONCLUSÃO}

Conseguir essa interação entre pacientes e profissionais desempenha um papel importante na melhoria da qualidade da assistência nas unidades de atenção à saúde, sendo um dos métodos alternativos que podem ser utilizados para desenvolver práticas de saúde integrais.

Assim, a extensão torna-se uma experiência que proporciona aos acadêmicos de enfermagem a participarem das atividades na comunidade juntamente com toda a população indígena, executando educação em saúde, na qual é um processo educativo em que propiciam a reflexão, baseada em estratégias que permitam mudanças de atitudes e comportamentos.

Os acadêmicos reconhecem a importância dessa vivencia para sua formação, tendo a perspectiva de que é um processo fundamental na atuação do profissional enfermeiro e toda equipe multiprofissional dentro do contexto associado a qualificação da saúde da mulher.

\section{DECLARAÇÃO DE INTERESSES}

Nós, autores deste artigo, declaramos que não possuímos conflitos de interesses de ordem financeira, comercial, político, acadêmico e pessoal.

\section{REFERÊNCIAS}

AUGUSTO, C. A. et al. Pesquisa Qualitativa: rigor metodológico no tratamento de teoria dos custos de transação em artigos apresentados nos congressos da Sober (2007-2011). RESR, Piracicaba-SP; Vol. 51, No 4, p.745-764, 2013.

COUTO, V. B. M. et al. "Além da Mama": o Cenário do Outubro Rosa no Aprendizado da Formação Médica. Revista Brasileira de Educação Médica. DOI: http://dx.doi.org/10.1590/198152712015 v41n1RB20160005

INCA. INSTITUTO NACIONAL DO CÂNCER, BRASIL. A situação do câncer de mama no Brasil: síntese de dados dos sistemas de informação. Rio de Janeiro, 2019.

OLIVEIRA, D.A.L. Educação em saúde no autocuidado contra o câncer de mama. REAID [Internet]. 11 abr.2019.

OLVIEIRA, M. M.; CORREIA, A. B.; FERREIRA, L. C. Ações do enfermeiro na deteç̧ão precoce do câncer de mama em mulheres no brasil: revisão integrativa. Revista Científica FacMais, Volume. XI, Número 4. dezembro. Ano 2017/2 Semestre. ISSN 2238- 8427.

SILVA, P. A.; RIUL, S.S. Câncer de mama: fatores de risco e detecção precoce. Rev Bras Enferm, Brasília 2011 nov-dez; 64(6): 1016-21. 


\section{Índice Remissivo}

A

Ações e serviços de saúde 15, 17

Ações multiprofissionais de educação em saúde 6, 22, 24

Alergia a proteína ao leite de vaca (aplv) 88

Anticoncepção pós-coito 95

Anticoncepcional de emergência 95

Aplicativos relacionados à saúde 134, 135, 139

Assistência pré-natal 80

Atenção básica 15, 19, 25, 27, 35, 37, 84, 100, 118, 120, 121, 122

Atenção básica no enfrentamento à covid-19 15

Atenção primária à saúde 15, 22, 23, 35, 36, 41, 49, 120, 124

Atendimento à população 22, 33

Atividades educativas sobre aplv 126

Atopia 88

Autocuidado 18, 44, 62, 64, 66, 73, 76, 123

Autoexame das mamas 39, 42

Avanços tecnológicos 113, 134

$\mathrm{C}$

Câncer de colo uterino 80

Câncer de mama 39, 40, 41, 42, 43, 44, 83, 85

Cárie dentária 71, 72, 73, 74, 75, 77

Cárie dentária na infância 71,73

Cárie na primeira infância (cpi) 71

Ciclo reprodutivo feminino 95, 96, 97, 98, 99

Climatério 40, 80, 82, 84

Comportamento contraceptivo 95

Comportamento sexual dos universitários 95

Contracepção 80,84

Covid-19 7, 12, 14, 15, 16, 17, 19, 20, 31, 32, 36, 45, 46, 47, 48, 49, 50, 52, 53, 54, 57, 60, 61, 92, $115,119,120,122,123,124,125,126,127,128,129$

Crianças 52, 54, 55, 57, 58, 59, 60, 71, 72, 73, 74, 75, 76, 77, 78, 79, 89, 91, 126, 127, 128, 131

Cuidado 28, 31, 62, 65, 93

Cuidados com a saúde $57,73,74,80,84$

Curva epidêmica 15,16 
Diagnóstico precoce à covid-19 15, 17

Direitos reprodutivos 80,83

Doença infecciosa $16,45,46$

Doença multifatorial 71

Doenças pulmonares crônicas 134

Doenças respiratórias crônicas 134

\section{E}

Educação em saúde 20, 22, 44, 53, 55, 71

Educação em saúde bucal 71, 72, 73, 75, 76, 77, 79

Educação em saúde para crianças 52

Educação infantil sobre a aplv 88

Educação interprofissional 22

Ensino e serviço 15

Equipe multiprofissional 9, 15, 62

Estado de calamidade pública 118

F

Falta de acesso às informações 102

Fisioterapia 118, 120, 122

Fisioterapia na atenção básica de saúde 118, 120

G

Gestão em saúde 15, 19

Gravidez indesejada 95, 98, 99, 100

$\mathrm{H}$

Hábitos nocivos 80,81

Hábitos saudáveis $80,81,82$

Hipersensibilidade a leite 127

Hipersensibilidade tipo i 88

Horários de atuação da equipe 15,18

I

Infecções por coronavírus 53

Interface usuário e aplicativos relacionados à saúde 134

Intervenção da telerreabilitação 118 
Leishmania 45, 46, 50

Leishmaniose 45, 46, 47, 49, 50

Leishmaniose cutânea 45

Linha de frente 15, 16, 32, 118

M

Medidas de biossegurança 52, 54, 57

M-health 134, 135, 138, 140

Mídias sociais 127

Mobile health 135, 140, 141

Mudança de hábitos 52, 75

$\mathrm{N}$

Neoplasia 62, 63, 65

$\mathrm{O}$

Obtenção de ists 95, 100

Oncologia 62, 65, 68

Orientação populacional e comunitária 15, 16

$\mathrm{P}$

Parasitas 45, 46

Período pandêmico 52, 56, 57, 58

Prevenção de doenças 53

Prevenção de ist/hiv 80

Prevenção do câncer de mama 39

Processo de saúde-doença 22, 32

Promoção à saúde 6,118

Q

Qualificação dos profissionais de saúde 22, 23

$\mathrm{R}$

Reação de hipersensibilidade 88

Reações alérgicas 126, 127

Recomendações sanitárias 15, 118

Rede hospitalar 15, 16

Redes de comunicação digital 102

Redes de internet 102, 104

Rede social instagram ${ }^{\circledR} 126$ 
Residência multiprofissional em saúde 15, 22, 23, 24, 35, 36

Residência multiprofissional em saúde da família 15

$\mathrm{S}$

Saúde bucal 71, 72, 73, 74, 75, 76, 78, 79

Saúde da família 6, 15, 22, 24, 25, 35, 36, 42, 118, 121, 122

Saúde da mulher 39, 40, 44, 80, 81

Saúde indígena 39

Saúde mental 28, 30, 31, 37, 53, 56, 57, 59, 60, 102, 103, 104, 106, 107, 108, 110, 111, 114, 115

Saúde pública 39, 40, 41, 125, 128, 135

Serviço social 22

Sistemas de saúde no brasil e no mundo 15, 16

Sistema único de saúde (sus) 15, 16, 17, 22, 23, 34, 35, 42, 91, 108

Smartphone applications 135

$\mathrm{T}$

Tecnologias móveis 134, 136

Tecnológicas de saúde 102

Teleconsulta 15, 121, 124

Telerreabilitação 15,118

Terapia medicamentosa 62,66

Terapia ocupacional 118,120

Tratamento intralesional de lcl 45, 47, 49

Tratamento oncológico 62, 63, 64, 65

$\mathrm{U}$

Unidades de saúde da família 22

Uso de máscara e álcool em gel 15

Utilização de aplicativos relacionados a promoção da saúde 134

V

Viabilidade do uso de m-health apps 134

Vínculo médico-paciente 45, 48

Violência contra a mulher 80,82 


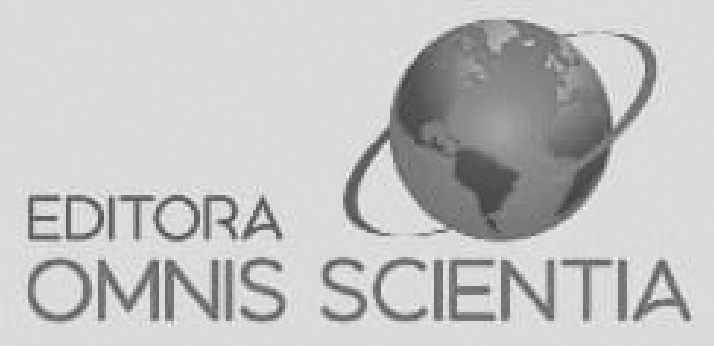

editoraomnisscientia@gmail.com M https://editoraomnisscientia.com.br/ @editora_omnis_scientia (0) https://www.facebook.com/omnis.scientia.9 $\subsetneq$ +55 (87) 9656-3565 (C) 


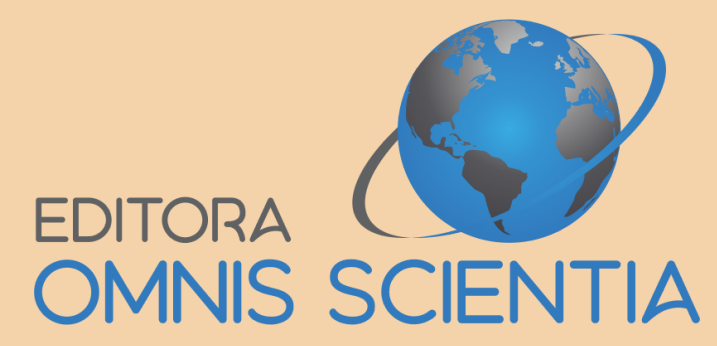

editoraomnisscientia@gmail.com M https://editoraomnisscientia.com.br/

@editora_omnis_scientia (0)

https://www.facebook.com/omnis.scientia.9 $\bigoplus$ +55 (87) 9656-3565 